\title{
Effect of Nitrogenous Fertilizer and Insecticides on Incidence of Leafhopper, Amrasca biguttula biguttula (Ishida) in Kharif Okra
}

\author{
Naziya P. Pathan ${ }^{1 *}$, T.M. Bharpoda ${ }^{1}$, D.B. Sisodiya ${ }^{2}$ and N.M. Gohel ${ }^{2}$ \\ ${ }^{1}$ Department of Entomology, B. A. Collage of Agriculture, Agricultural Research Station, \\ Arnej, Gujarat, India \\ ${ }^{2}$ Department of Plant Pathology, B. A. Collage of Agriculture, Anand Agricultural University, \\ Anand- 388110, Gujarat, India \\ *Corresponding author
}

\begin{tabular}{|c|c|}
\hline & A B S T R A C T \\
\hline Keywords & \multirow{4}{*}{$\begin{array}{l}\text { Impact of nitrogenous fertilizer and insecticides on incidence of leafhopper, } \\
\text { Amrasca biguttula biguttula in okra was carried out at Agronomy Farm, B. A. } \\
\text { College of Agriculture, Anand Agricultural University, Anand during Kharif, } \\
\text { 2015. Results of pooled over three sprays during kharif, } 2015 \text { revealed that } \\
\text { flonicamid (0.65) was found significantly superior than rest of the insecticides. } \\
\text { The second best insecticide was dinotefuran (1.49). Thiamethoxam }(2.23) \text { and } \\
\text { dimethoate (2.52) were at par with each other. While different doses of } \\
\text { nitrogenous fertilizer did not have significant impact on population development } \\
\text { of leafhopper. The interaction between different doses of nitrogenous fertilizer and } \\
\text { insecticides was found non-significant. It indicated that there was no any } \\
\text { significant impact of nitrogenous fertilizers on efficacy of insecticides on } \\
\text { leafhopper in okra. }\end{array}$} \\
\hline $\begin{array}{l}\text { Flonicamid, } \\
\text { Thiamethoxam, } \\
\text { Dinotefuran, } \\
\text { Dimethoate and } \\
\text { Okra. }\end{array}$ & \\
\hline Article Info & \\
\hline $\begin{array}{l}\text { Accepted: } \\
\text { 07 September } 2017 \\
\text { Available Online: } \\
10 \text { October } 2017\end{array}$ & \\
\hline
\end{tabular}

\section{Introduction}

Okra (Abelmoschus esculentus L. Moench) is the only vegetable crop of significance in the Malvaceae family. The average production of okra in India is about 57.84 lakh tones and productivity 11.6 tones/ha (Pal et al., 2013). In India, it is cultivated in 532.7 lakh hectares with a production of 6346.4 lakh MT and with the productivity of 11.9 MT/ha during 20132014 (Anon., 2014). One of the major constraints for okra production is heavy infestations caused by several insect pests which not only exert quantitative loss but also caused qualitative loss to the crop.
As many as, 72 insect species have been recorded on okra (Pal et al., 2013). Among sucking pests, leafhopper is the major pest, which cause a yield loss of about 40-56 per cent in okra (Krishnaiah, 1980). One to the infestation of this pest, there was a reduction of 49.8 and 45.1 per cent in height and number of leaves, respectively (Rawat and Sadhu, 1973). Plants become more succulent and having profuse growth due to the use of nitrogenous fertilizer beyond its recommended dose, which ultimately invite large number of sucking pests. Under this 
circumstance, farmers move to use insecticides indiscriminately with higher dose. Though conventional insecticides offers good control, their residue levels are high besides causing adverse effect like resurgence and resistance (Sridharan et al., 2015). In this context, it is important to evaluate the efficacy of newer molecules with novel mode of action to find out the viable option for sustainable management of sucking insect pests of okra. Therefore, with a view to study the impact of nitrogenous fertilizer and insecticides on incidence of leaf hopper, present experiment was carried out.

\section{Materials and Methods}

\section{Details of experiment}

The experiment was conducted during kharif 2015 at Agronomy Farm, B. A. College of Agriculture, Anand Agricultural University, Anand. Okra variety GAO 5 was raised at 45 $\mathrm{X} 15 \mathrm{~cm}$ following standard agronomical practices. In order to study the effect of nitrogenous fertilizer and insecticides on incidence of leafhopper in okra, the experiment was laid out in a split plot design with three replications having plot size of 2.7 $\mathrm{x} 15.0 \mathrm{~m}$. All fertilizer doses were applied in different plots according to respective treatments. Fifty percent nitrogen (in urea form) and 100 per cent phosphorus and potash (in form of single super phosphate and murate of potash) were applied at the time of sowing, while remaining 50 per cent nitrogen was applied at 30 days after sowing followed by earthing up operation. The first spray of respective insecticides was given on initiation of pests and subsequently two sprays were followed on need based. Dimethoate $30 \mathrm{EC}$ $(0.03 \%)$, thiamethoxam $25 \mathrm{WG}(0.01 \%)$, flonicamid $50 \mathrm{WP}(0.015 \%)$ and dinotefuran 20 SG (0.008\%) was evaluated against leafhopper with three different nitrogenous fertilizer doses viz., $\mathrm{N}_{1}(50 \mathrm{~kg} / \mathrm{ha}), \mathrm{N}_{2}(100$ $\mathrm{kg} / \mathrm{ha}$ ) and $\mathrm{N}_{3}\left(150 \mathrm{~kg} / \mathrm{ha}\right.$ ) as well as $\mathrm{N}_{0}$ (no nitrogen).

\section{Method of recording observation of leafhopper}

The observations on population of leafhopper, A. biguttula biguttula on three leaves (top, middle and bottom canopy) from the five randomly selected plants were recorded from each plot. The observations were recorded at before spray and 1, 3, 5, 7 days after application of insecticides, respectively for each spray. The data obtained were statistically analyzed after following appropriate transformation.

The yield of okra fruit was recorded at periodical pickings as and when ready to harvest. Yield of healthy okra fruit were recorded periodically at and when ready to harvest from each sectors separately. Fourteen pickings were made and data on okra fruit yield were summed up for further statistical analysis.

\section{Results and Discussions}

\section{First spray}

The periodic data on leafhopper population was recorded after first spray during kharif, 2015 are presented in Table 1.

\section{Independent effect of insecticides (I)}

The leafhopper population was uniform in all the treatments before spray as treatment difference was non-significant. All the insecticides were significantly superior to untreated control up to 7 DAS. At 1 DAS, the highest effect was observed with the application of flonicamid $50 \mathrm{WP} 0.015 \%$ as it recorded significantly the lowest $(0.71$ leafhoppes/leaf) leafhopper population (Table 1). All the insecticidal treatments differed 
significantly with each other. Dinotefuran 20 SG $0.008 \%$ also recorded lower (2.56) population of leafhopper. Thiamethoxam 25 WP $0.01 \%$ (2.34) was found equally effective as dimethoate 30 EC $0.03 \%$ (3.62) in suppressing the leafhopper incidence in okra. More or less similar trend was observed at 3 and 5 DAS. At 7 DAS, flonicamid $50 \mathrm{WP}$ $0.015 \% \quad(0.33)$ was found significantly superior but remained at par with dinotefuran 20 SG $0.008 \%$ (0.44). The second better treatment was thiamethoxam $25 \mathrm{WP} 0.01 \%$ (1.69) followed by dimethoate $30 \mathrm{EC}$ at $0.03 \%$ (4.84). Among the treatments, the highest (4.84) population of leafhopper was observed in control plots.

Pooled over periods of mean data (Table 1) indicated that significantly the highest effectiveness was observed with application of flonicamid 50 WP $0.015 \%$ and recorded the lowest (0.97 leafhoppes/leaf) population. Dinotefuran 20 SG $0.008 \%$ (1.24) was also effective in reducing leafhopper population in okra by registered second best treatment. Thiamethoxam 25 WP $0.01 \% \quad$ (2.74) and dimethoate 30 EC $0.03 \%$ (2.81) were equally effective in reducing leafhopper population. Of the treatments, the highest (4.74) population of leafhopper was noticed in control plots.

\section{Independent effect of fertilizer $(\mathbf{N})$}

The effect of nitrogenous fertilizer at different doses on incidence of leafhopper is depicted in Table 1. A uniform population of pest was observed before application of treatments as differences among the treatments was nonsignificant. At 1 DAS, minimum population (2.42 leafhoppers/leaf) of leafhopper was observed in control (No fertilizer) treatment which was at par with $\mathrm{N}_{1}(50 \mathrm{~kg} / \mathrm{ha})$ i.e. 2.70 . The highest dose of fertilizer, $\mathrm{N}_{3}(150 \mathrm{~kg} / \mathrm{ha})$ had 3.42 leafhoppers per leaf and was at par with recommended dose, $\mathrm{N}_{2}(100 \mathrm{~kg} / \mathrm{ha})$. At 3
DAS, minimum population of leafhopper (1.90) was recorded in $\mathrm{N}_{0}$. The other treatments $\mathrm{N}_{1}, \mathrm{~N}_{2}$ and $\mathrm{N}_{3}$ were at par with each other and recorded 2.96, 2.36 and 2.74, respectively. More or less similar trend was observed at 5 and 7 DAS.

The pooled data over periods clearly indicated that minimum population of leafhopper was observed in $\mathrm{N}_{0}$ (1.66 leafhoppes/leaf) and it was at par with $\mathrm{N}_{2}(100 \mathrm{~kg} / \mathrm{ha})$. The highest dose of nitrogenous fertilizer $\left(\mathrm{N}_{3}\right)$ showed 2.49 leafhopper population. However, it was at par with $\mathrm{N}_{1}(50 \mathrm{~kg} / \mathrm{ha})$.

Interactive effect of insecticide and fertilizer $(\mathbf{I} \times \mathbf{N})$

The interactive effect of insecticides with different dose of nitrogenous fertilizer $(\mathrm{I} \times \mathrm{N})$ on okra crop did not show any significant impact on population of leafhopper as the results are non-significant (Table 1).

\section{Second spray}

The periodical data on leafhopper, $A$. biguttula biguttula recorded during kharif, 2015 after second spray are presented in Table 2.

\section{Independent effect of insecticides (I)}

The population of leafhopper was homogenous in all the treatments before spray as treatment difference was non-significance. All the evaluated insecticides were significantly superior to untreated control up to 7 DAS. At 1 DAS, significantly the lowest (1.87 leafhoppers/leaf) population of leafhopper was recorded in plots treated with flonicamid 50 WP $0.015 \%$ and found most effective insecticidal treatment. Dinotefuran 20 SG $0.008 \%$ and thiamethoxam 25 WP $0.01 \%$ were equally effective in reducing leafhopper population as they are at par with 
each other by registering 2.78 and 3.07, respectively. Dimethoate 30 EC $0.03 \%$ was found least effective against leafhoppers in okra. Of the treatments, control plots registered the highest (5.45) number of leafhopper. At 3 DAS, significantly the lowest (0.48) leafhopper incidence was observed in plots treated with flonicamid 50 WP $0.015 \%$.

All the treatments differ significantly with each other. Dinotefuran 20 SG 0.008\% (1.43) proved to be second best insecticide followed by thiamethoxam 25 WP $0.01 \% \quad$ (2.06). Dimethoate 30 EC $0.03 \%$ (3.03) was the least effective among different insecticidal treatments. The untreated control plots recorded higher (5.45) leafhopper population in okra. At 5 DAS, among the evaluated insecticides, significantly higher (0.42) effect was recorded in flonicamid 50 WP $0.015 \%$. Dinotefuran 20 SG $0.008 \% \quad$ (1.09) and thiamethoxam 25 WP $0.01 \%$ (1.11) were equally effective in reducing leafhopper population as they were at par with each other. Dimethoate $30 \mathrm{EC}$ at $0.03 \%$ proved to be least effective as it recorded higher (1.60) leafhopper population on okra. The untreated control plot registered the higher (5.40) number of leafhoppers. More or less, same trend of treatment effect was observed at 7 DAS.

Pooled over periods of mean data (Table 2) indicated that the lowest $(0.71$ leafhoppers/leaf) leafhopper population was found in the plots treated with flonicamid 50 WP $0.015 \%$ (0.71) and proved to be most effective among all the evaluated insecticides. Dinotefuran 20 SG $0.008 \%$ and thiamethoxam 25 WP $0.01 \%$ were found equally effective in suppressing the leafhopper population in okra. Dimethoate 30 EC at $0.03 \%$ was found less effective insecticide as it recorded significantly higher (2.32) leafhopper population.

\section{Independent effect of fertilizer (N)}

The data on impact of nitrogenous fertilizer at different doses on incidence of leafhopper are presented in Table 2. All doses of nitrogenous fertilizer did not show any significant effect on population built up of leafhoppers.

\section{Interactive effect of insecticide and fertilizer $(\mathbf{I} \times \mathbf{N})$}

The interactive effect of insecticides with different dose of nitrogenous fertilizer on okra crop showed non-significant impact on population of $A$. biguttula biguttula as per the data presented in Table 2.

The interaction $\mathrm{I} \times \mathrm{P}$ was found significant indicating the inconsistence performance of insecticides at different periods.

Like-wise, interaction $\mathrm{N} \times \mathrm{P}$ was found nonsignificant which indicates the consistence performance of various doses of nitrogenous fertilizer at varying periods on leafhopper in okra.

\section{Third spray}

The periodical data on leafhopper A. biguttula biguttula recorded during kharif, 2015, $3^{\text {rd }}$ sprays are presented in Table 3.

\section{Independent effect of insecticides (I)}

The leafhopper population was uniform in all the treatments before spray as treatment difference was non-significant. At 1 DAS, the highest effect was observed with application of flonicamid 50 WP $0.015 \%$ as it recorded significantly the lowest (1.78 leafhoppers/leaf) leafhopper population (Table 3). Dinotefuran 20 SG $0.008 \%$, thiamethoxam $25 \mathrm{WP} 0.01 \%$ and dimethoate 30 EC $0.03 \%$ were at par and registered 3.70, 3.95 and 3.77 , respectively. 
Table.1 Impact of insecticides and nitrogenous fertilizer on incidence of leafhopper, A. biguttula biguttula in okra after $1^{\text {st }}$ spray (kharif, 2015)

\begin{tabular}{|c|c|c|c|c|c|c|c|}
\hline \multirow{2}{*}{\multicolumn{2}{|c|}{$\begin{array}{c}\text { Treatment } \\
\text { Insecticides (I) }\end{array}$}} & \multirow{2}{*}{$\begin{array}{c}\text { Before } \\
\text { spray }\end{array}$} & \multicolumn{4}{|c|}{ Leafhoppers/leaf days after spray (DAS) } & \multirow[t]{2}{*}{ Mean } \\
\hline & & & 1 DAS & 3 DAS & 5 DAS & 7 DAS & \\
\hline \multicolumn{2}{|r|}{$\mathbf{I}_{\mathbf{0}}$} & $\begin{array}{c}2.32 \\
(4.88)\end{array}$ & $\begin{array}{l}2.26 \mathrm{~d} \\
(4.61)\end{array}$ & $\begin{array}{l}2.28 \mathrm{~d} \\
(4.70)\end{array}$ & $\begin{array}{l}2.30 \mathrm{~d} \\
(4.79)\end{array}$ & $\begin{array}{l}2.31 \mathrm{c} \\
(4.84)\end{array}$ & $\begin{array}{l}2.29 \mathrm{~d} \\
(4.74)\end{array}$ \\
\hline \multicolumn{2}{|r|}{$\mathbf{I}_{\mathbf{1}}$} & $\begin{array}{c}2.22 \\
(4.43)\end{array}$ & $\begin{array}{l}2.03 c \\
(3.62)\end{array}$ & $\begin{array}{l}1.96 \mathrm{c} \\
(3.34)\end{array}$ & $\begin{array}{l}1.75 \mathrm{c} \\
(2.56)\end{array}$ & $\begin{array}{l}1.48 \mathrm{~b} \\
(1.69)\end{array}$ & $\begin{array}{l}1.82 \mathrm{c} \\
(2.81)\end{array}$ \\
\hline \multicolumn{2}{|r|}{$\mathbf{I}_{2}$} & $\begin{array}{c}2.36 \\
(5.07)\end{array}$ & $\begin{array}{l}2.20 \mathrm{~cd} \\
(4.34)\end{array}$ & $\begin{array}{l}1.98 \mathrm{c} \\
(3.42)\end{array}$ & $\begin{array}{l}1.62 \mathrm{c} \\
(2.12)\end{array}$ & $\begin{array}{l}1.38 \mathrm{~b} \\
(1.40)\end{array}$ & $\begin{array}{l}1.80 \mathrm{c} \\
(2.74)\end{array}$ \\
\hline \multicolumn{2}{|r|}{$\mathbf{I}_{3}$} & $\begin{array}{c}2.29 \\
(4.74)\end{array}$ & $\begin{array}{r}1.10 \mathrm{a} \\
(0.71)\end{array}$ & $\begin{array}{l}0.95 \mathrm{a} \\
(0.40)\end{array}$ & $\begin{array}{l}0.92 \mathrm{a} \\
(0.35)\end{array}$ & $\begin{array}{r}0.91 \mathrm{a} \\
(0.33)\end{array}$ & $\begin{array}{r}0.97 \mathrm{a} \\
(0.44)\end{array}$ \\
\hline \multicolumn{2}{|r|}{$\mathbf{I}_{4}$} & $\begin{array}{c}2.24 \\
(4.52)\end{array}$ & $\begin{array}{l}1.75 b \\
(2.56)\end{array}$ & $\begin{array}{l}1.43 \mathrm{~b} \\
(1.54)\end{array}$ & $\begin{array}{l}1.12 \mathrm{~b} \\
(0.75)\end{array}$ & $\begin{array}{r}0.97 \mathrm{a} \\
(0.44)\end{array}$ & $\begin{array}{l}1.32 b \\
(1.24)\end{array}$ \\
\hline \multicolumn{2}{|c|}{$\begin{array}{c}\text { S. Em. } \pm \\
\text { C. D. at } 5 \%\end{array}$} & $\begin{array}{l}0.09 \\
\mathrm{NS}\end{array}$ & $\begin{array}{l}0.06 \\
0.18\end{array}$ & $\begin{array}{l}0.06 \\
0.18\end{array}$ & $\begin{array}{l}0.04 \\
0.13\end{array}$ & $\begin{array}{l}0.06 \\
0.18\end{array}$ & $\begin{array}{l}0.05 \\
0.16\end{array}$ \\
\hline \multicolumn{2}{|c|}{ C. V. \% } & 13.10 & 10.39 & 11.82 & 9.59 & 14.43 & 20.91 \\
\hline $\mathbf{I} \times \mathbf{P}$ & $\begin{array}{c}\text { S. Em. } \pm \\
\text { C. D. at } 5 \%\end{array}$ & & & & & & $\begin{array}{l}0.03 \\
0.10\end{array}$ \\
\hline \multirow{2}{*}{\multicolumn{2}{|c|}{ Nitrogenous fertilizer $(\mathbf{N})$}} & Before & \multicolumn{4}{|c|}{ Leafhoppers/leaf days after spray (DAS) } & \multirow[t]{2}{*}{ Mean } \\
\hline & & spray & 1 DAS & 3 DAS & 5 DAS & 7 DAS & \\
\hline \multicolumn{2}{|r|}{$\mathbf{N}_{\mathbf{0}}$} & $\begin{array}{c}2.06 \\
(3.74)\end{array}$ & $\begin{array}{l}1.71 \mathrm{a} \\
(2.42)\end{array}$ & $\begin{array}{l}1.55 \mathrm{a} \\
(1.90)\end{array}$ & $\begin{array}{l}1.38 \mathrm{a} \\
(1.40)\end{array}$ & $\begin{array}{l}1.26 \mathrm{ab} \\
(1.09)\end{array}$ & $\begin{array}{l}1.47 \mathrm{a} \\
(1.66)\end{array}$ \\
\hline \multicolumn{2}{|r|}{$\mathbf{N}_{1}$} & $\begin{array}{c}2.49 \\
(5.70)\end{array}$ & $\begin{array}{l}2.06 \mathrm{c} \\
(3.74)\end{array}$ & $\begin{array}{l}1.86 \mathrm{~b} \\
(2.96)\end{array}$ & $\begin{array}{l}1.64 \mathrm{~b} \\
(2.19)\end{array}$ & $\begin{array}{l}1.49 \mathrm{c} \\
(1.72)\end{array}$ & $\begin{array}{l}1.76 \mathrm{c} \\
(2.60)\end{array}$ \\
\hline \multicolumn{2}{|r|}{$\mathbf{N}_{2}$} & $\begin{array}{c}2.23 \\
(4.47)\end{array}$ & $\begin{array}{l}1.79 \mathrm{ab} \\
(2.70)\end{array}$ & $\begin{array}{l}1.69 \mathrm{ab} \\
(2.36)\end{array}$ & $\begin{array}{l}1.53 \mathrm{ab} \\
(1.84)\end{array}$ & $\begin{array}{l}1.38 \mathrm{bc} \\
(1.40)\end{array}$ & $\begin{array}{l}1.60 \mathrm{ab} \\
(2.06)\end{array}$ \\
\hline \multicolumn{2}{|r|}{$\mathbf{N}_{3}$} & $\begin{array}{c}2.37 \\
(5.12) \\
\end{array}$ & $\begin{array}{l}1.98 \mathrm{bc} \\
(3.42)\end{array}$ & $\begin{array}{l}1.80 \mathrm{~b} \\
(2.74)\end{array}$ & $\begin{array}{l}1.62 \mathrm{~b} \\
(2.12)\end{array}$ & $\begin{array}{r}1.15 \mathrm{a} \\
(0.82) \\
\end{array}$ & $\begin{array}{l}1.73 b c \\
(2.49)\end{array}$ \\
\hline \multicolumn{2}{|c|}{$\begin{array}{c}\text { S. Em. } \pm \\
\text { C. D. at } 5 \%\end{array}$} & $\begin{array}{l}0.08 \\
\mathrm{NS}\end{array}$ & $\begin{array}{l}0.07 \\
0.19\end{array}$ & $\begin{array}{l}0.06 \\
0.17\end{array}$ & $\begin{array}{l}0.06 \\
0.16\end{array}$ & $\begin{array}{l}0.05 \\
0.14\end{array}$ & $\begin{array}{l}0.05 \\
0.15\end{array}$ \\
\hline \multicolumn{2}{|c|}{ C. V.\% } & 12.97 & 13.73 & 13.4 & 13.69 & 13.60 & 24.24 \\
\hline $\mathbf{N} \times \mathbf{P}$ & $\begin{array}{c}\text { S. Em. } \pm \\
\text { C. D. at } 5 \%\end{array}$ & & & & & & $\begin{array}{c}0.03 \\
\mathrm{NS}\end{array}$ \\
\hline $\mathbf{I} \times \mathbf{N}$ & $\begin{array}{c}\text { S. Em. } \pm \\
\text { C. D. at } 5 \%\end{array}$ & & & & & & $\begin{array}{l}0.12 \\
\mathrm{NS}\end{array}$ \\
\hline
\end{tabular}

Note:

1. Figures in parentheses are retransformed values; those outside are $\sqrt{\mathrm{X}+0.5}$ value

2. Treatment means with letter (s) in common are not significant at $5 \%$ level of significance

3. Insecticide: $\mathrm{I}_{0}=$ Control (No spray), $\mathrm{I}_{1}=$ Dimethoate $30 \mathrm{EC}, \mathrm{I}_{2}=$ Thiamethoxam $25 \mathrm{WG}, \mathrm{I}_{3}=$ Flonicamid $50 \mathrm{WP}$ and $\mathrm{I}_{4}=$

Dinotefuran 20 SG

4. Fertilizer: $\mathrm{N}_{0}=0 \mathrm{~kg} / \mathrm{ha}, \mathrm{N}_{1}=50 \mathrm{~kg} / \mathrm{ha}, \mathrm{N}_{2}=100 \mathrm{~kg} / \mathrm{ha}$ and $\mathrm{N}_{3}=150 \mathrm{~kg} / \mathrm{ha}$

5. Okra variety: Gujarat Anand Okra-5 
Table.2 Impact of insecticides and nitrogenous fertilizer on incidence of leafhopper,

A. biguttula biguttula in okra after $2^{\text {nd }}$ spray (kharif, 2015)

\begin{tabular}{|c|c|c|c|c|c|c|c|}
\hline \multirow{2}{*}{\multicolumn{2}{|c|}{$\frac{\text { Treatment }}{\text { Insecticides (I) }}$}} & \multirow{2}{*}{$\begin{array}{l}\text { Before } \\
\text { spray }\end{array}$} & \multicolumn{4}{|c|}{ Leafhoppers/leaf days after spray (DAS) } & \multirow[t]{2}{*}{ Mean } \\
\hline & & & 1 DAS & 3 DAS & 5 DAS & 7 DAS & \\
\hline \multicolumn{2}{|c|}{$\mathbf{I}_{\mathbf{0}}$} & $\begin{array}{c}2.43 \\
(5.40) \\
\end{array}$ & $\begin{array}{l}2.44 d \\
(5.45) \\
\end{array}$ & $\begin{array}{l}2.44 \mathrm{e} \\
(5.45)\end{array}$ & $\begin{array}{l}2.43 \mathrm{~d} \\
(5.40) \\
\end{array}$ & $\begin{array}{l}2.45 \mathrm{~d} \\
(5.50) \\
\end{array}$ & $\begin{array}{l}2.44 d \\
(5.45)\end{array}$ \\
\hline \multicolumn{2}{|r|}{$\mathbf{I}_{1}$} & $\begin{array}{c}2.49 \\
(5.70) \\
\end{array}$ & $\begin{array}{l}2.24 c \\
(4.52) \\
\end{array}$ & $\begin{array}{l}1.88 \mathrm{~d} \\
(3.03) \\
\end{array}$ & $\begin{array}{l}1.45 \mathrm{c} \\
(1.60) \\
\end{array}$ & $\begin{array}{l}1.16 \mathrm{c} \\
(0.85) \\
\end{array}$ & $\begin{array}{l}1.68 \mathrm{c} \\
(2.32) \\
\end{array}$ \\
\hline \multicolumn{2}{|r|}{$\mathbf{I}_{2}$} & $\begin{array}{c}2.51 \\
(5.80) \\
\end{array}$ & $\begin{array}{l}1.89 \mathrm{~b} \\
(3.07) \\
\end{array}$ & $\begin{array}{l}1.60 \mathrm{c} \\
(2.06) \\
\end{array}$ & $\begin{array}{l}1.27 \mathrm{~b} \\
(1.11)\end{array}$ & $\begin{array}{l}1.05 \mathrm{bc} \\
(0.60)\end{array}$ & $\begin{array}{l}1.45 b \\
(1.60)\end{array}$ \\
\hline \multicolumn{2}{|r|}{$\mathbf{I}_{3}$} & $\begin{array}{c}2.46 \\
(5.55)\end{array}$ & $\begin{array}{l}1.54 \mathrm{a} \\
(1.87)\end{array}$ & $\begin{array}{l}0.99 a \\
(0.48)\end{array}$ & $\begin{array}{l}0.96 \mathrm{a} \\
(0.42)\end{array}$ & $\begin{array}{l}0.92 \mathrm{a} \\
(0.35)\end{array}$ & $\begin{array}{l}1.10 \mathrm{a} \\
(0.71)\end{array}$ \\
\hline \multicolumn{2}{|r|}{$\mathbf{I}_{4}$} & $\begin{array}{c}2.39 \\
(5.21) \\
\end{array}$ & $\begin{array}{l}1.81 \mathrm{~b} \\
(2.78) \\
\end{array}$ & $\begin{array}{l}1.39 \mathrm{~b} \\
(1.43) \\
\end{array}$ & $\begin{array}{l}1.26 \mathrm{~b} \\
(1.09) \\
\end{array}$ & $\begin{array}{l}1.02 \mathrm{ab} \\
(0.54) \\
\end{array}$ & $\begin{array}{l}1.37 \mathrm{~b} \\
(1.38) \\
\end{array}$ \\
\hline \multicolumn{2}{|c|}{$\begin{array}{c}\text { S. Em. } \pm \\
\text { C. D. at } 5 \%\end{array}$} & $\begin{array}{c}0.08 \\
\mathrm{NS}\end{array}$ & $\begin{array}{l}0.06 \\
0.18\end{array}$ & $\begin{array}{l}0.05 \\
0.15\end{array}$ & $\begin{array}{l}0.06 \\
0.17\end{array}$ & $\begin{array}{l}0.04 \\
0.13\end{array}$ & $\begin{array}{l}0.04 \\
0.14\end{array}$ \\
\hline \multicolumn{2}{|c|}{ C. V. \% } & 10.87 & 10.25 & 9.97 & 12.45 & 10.87 & 18.29 \\
\hline $\mathbf{I} \times \mathbf{P}$ & $\begin{array}{c}\text { S. Em. } \pm \\
\text { C. D. at } 5 \%\end{array}$ & & & & & & $\begin{array}{l}0.03 \\
0.10\end{array}$ \\
\hline \multirow{2}{*}{\multicolumn{2}{|c|}{ Nitrogenous fertilizer $(\mathbf{N})$}} & Before & \multicolumn{4}{|c|}{ Leafhoppers/leaf days after spray (DAS) } & \multirow[t]{2}{*}{ Mean } \\
\hline & & spray & 1 DAS & 3 DAS & 5 DAS & 7 DAS & \\
\hline \multicolumn{2}{|r|}{$\mathbf{N}_{0}$} & $\begin{array}{c}2.49 \\
(5.70) \\
\end{array}$ & $\begin{array}{c}2.00 \\
(3.50) \\
\end{array}$ & $\begin{array}{c}1.68 \\
(2.32) \\
\end{array}$ & $\begin{array}{c}1.51 \\
(1.78) \\
\end{array}$ & $\begin{array}{c}1.34 \\
(1.30) \\
\end{array}$ & $\begin{array}{c}1.63 \\
(2.16)\end{array}$ \\
\hline \multicolumn{2}{|r|}{$\mathbf{N}_{1}$} & $\begin{array}{c}2.46 \\
(5.55) \\
\end{array}$ & $\begin{array}{c}1.94 \\
(3.26) \\
\end{array}$ & $\begin{array}{c}1.65 \\
(2.22) \\
\end{array}$ & $\begin{array}{c}1.47 \\
(1.66) \\
\end{array}$ & $\begin{array}{c}1.33 \\
(1.27) \\
\end{array}$ & $\begin{array}{c}1.60 \\
(2.06) \\
\end{array}$ \\
\hline \multicolumn{2}{|r|}{$\mathbf{N}_{2}$} & $\begin{array}{c}2.43 \\
(5.40) \\
\end{array}$ & $\begin{array}{c}1.97 \\
(3.38) \\
\end{array}$ & $\begin{array}{c}1.62 \\
(2.12) \\
\end{array}$ & $\begin{array}{c}1.42 \\
(1.52) \\
\end{array}$ & $\begin{array}{c}1.26 \\
(1.09) \\
\end{array}$ & $\begin{array}{c}1.57 \\
(1.96)\end{array}$ \\
\hline \multicolumn{2}{|r|}{$\mathbf{N}_{3}$} & $\begin{array}{c}2.44 \\
(5.45) \\
\end{array}$ & $\begin{array}{c}2.02 \\
(3.58) \\
\end{array}$ & $\begin{array}{c}1.68 \\
(2.32) \\
\end{array}$ & $\begin{array}{c}1.49 \\
(1.72) \\
\end{array}$ & $\begin{array}{c}1.35 \\
(1.32) \\
\end{array}$ & $\begin{array}{r}1.64 \\
(2.19) \\
\end{array}$ \\
\hline \multicolumn{2}{|c|}{$\begin{array}{c}\text { S. Em. } \pm \\
\text { C. D. at } 5 \%\end{array}$} & $\begin{array}{c}0.08 \\
\mathrm{NS}\end{array}$ & $\begin{array}{l}0.07 \\
\text { NS }\end{array}$ & $\begin{array}{l}0.04 \\
\text { NS }\end{array}$ & $\begin{array}{l}0.03 \\
\text { NS }\end{array}$ & $\begin{array}{l}0.03 \\
\text { NS }\end{array}$ & $\begin{array}{l}0.04 \\
\mathrm{NS}\end{array}$ \\
\hline \multicolumn{2}{|c|}{ C. V. \% } & 14.27 & 12.9 & 9.79 & 8.14 & 8.79 & 17.00 \\
\hline $\mathbf{N} \times \mathbf{P}$ & $\begin{array}{c}\text { S. Em. } \pm \\
\text { C. D. at } 5 \%\end{array}$ & & & & & & $\begin{array}{c}0.03 \\
\mathrm{NS}\end{array}$ \\
\hline $\mathbf{I} \times \mathbf{N}$ & $\begin{array}{c}\text { S. Em. } \pm \\
\text { C. D. at } 5 \%\end{array}$ & & & & & & $\begin{array}{l}0.08 \\
\mathrm{NS}\end{array}$ \\
\hline
\end{tabular}

\section{Note:}

1. Figures in parentheses are retransformed values; those outside are $\sqrt{\mathrm{X}+0.5}$ value

2. Treatment means with letter (s) in common are not significant at $5 \%$ level of significance

3. Insecticide: $\mathrm{I}_{0}=$ Control (No spray), $\mathrm{I}_{1}=$ Dimethoate $30 \mathrm{EC}, \mathrm{I}_{2}=$ Thiamethoxam $25 \mathrm{WG}, \mathrm{I}_{3}=$ Flonicamid $50 \mathrm{WP}$ and $\mathrm{I}_{4}=$

Dinotefuran 20 SG

4. Fertilizer: $\mathrm{N}_{0}=0 \mathrm{~kg} / \mathrm{ha}, \mathrm{N}_{1}=50 \mathrm{~kg} / \mathrm{ha}, \mathrm{N}_{2}=100 \mathrm{~kg} / \mathrm{ha}$ and $\mathrm{N}_{3}=150 \mathrm{~kg} / \mathrm{ha}$

5. Okra variety: Gujarat Anand Okra-5 
Table.3 Impact of insecticides and nitrogenous fertilizer on incidence of leafhopper,

A. biguttula biguttula in okra after $3^{\text {rd }}$ spray (kharif, 2015)

\begin{tabular}{|c|c|c|c|c|c|c|c|}
\hline \multirow{2}{*}{\multicolumn{2}{|c|}{$\begin{array}{c}\text { Treatment } \\
\text { Insecticides (I) }\end{array}$}} & \multirow{2}{*}{$\begin{array}{c}\text { Before } \\
\text { spray }\end{array}$} & \multicolumn{4}{|c|}{ Leafhoppers/leaf days after spray (DAS) } & \multirow[t]{2}{*}{ Mean } \\
\hline & & & 1 DAS & 3 DAS & 5 DAS & 7 DAS & \\
\hline \multicolumn{2}{|r|}{$\mathbf{I}_{\mathbf{0}}$} & $\begin{array}{c}2.40 \\
(5.26)\end{array}$ & $\begin{array}{l}2.40 \mathrm{c} \\
(5.26)\end{array}$ & $\begin{array}{l}2.42 \mathrm{c} \\
(5.36)\end{array}$ & $\begin{array}{l}2.44 d \\
(5.45)\end{array}$ & $\begin{array}{l}2.21 \mathrm{c} \\
(4.38)\end{array}$ & $\begin{array}{l}2.37 \mathrm{c} \\
(5.12)\end{array}$ \\
\hline \multicolumn{2}{|r|}{$\mathbf{I}_{1}$} & $\begin{array}{c}2.39 \\
(5.21) \\
\end{array}$ & $\begin{array}{l}2.09 \mathrm{~b} \\
(3.87) \\
\end{array}$ & $\begin{array}{l}1.93 \mathrm{~b} \\
(3.22)\end{array}$ & $\begin{array}{l}1.53 \mathrm{bc} \\
(1.84)\end{array}$ & $\begin{array}{l}1.27 \mathrm{~b} \\
(1.11)\end{array}$ & $\begin{array}{l}1.71 b \\
(2.42)\end{array}$ \\
\hline \multicolumn{2}{|r|}{$\mathbf{I}_{2}$} & $\begin{array}{c}2.33 \\
(4.93)\end{array}$ & $\begin{array}{l}2.11 \mathrm{~b} \\
(3.95)\end{array}$ & $\begin{array}{l}1.91 \mathrm{~b} \\
(3.15)\end{array}$ & $\begin{array}{l}1.62 \mathrm{c} \\
(2.12)\end{array}$ & $\begin{array}{l}1.22 \mathrm{~b} \\
(0.99)\end{array}$ & $\begin{array}{l}1.71 \mathrm{~b} \\
(2.42)\end{array}$ \\
\hline \multicolumn{2}{|r|}{$\mathbf{I}_{3}$} & $\begin{array}{c}2.30 \\
(4.79) \\
\end{array}$ & $\begin{array}{r}1.51 \mathrm{a} \\
(1.78) \\
\end{array}$ & $\begin{array}{l}1.20 \mathrm{a} \\
(0.94) \\
\end{array}$ & $\begin{array}{l}0.95 \mathrm{a} \\
(0.40) \\
\end{array}$ & $\begin{array}{l}0.92 \mathrm{a} \\
(0.35) \\
\end{array}$ & $\begin{array}{l}1.15 \mathrm{a} \\
(0.82)\end{array}$ \\
\hline \multicolumn{2}{|r|}{$\mathbf{I}_{4}$} & $\begin{array}{c}2.29 \\
(4.74)\end{array}$ & $\begin{array}{l}2.05 b \\
(3.70)\end{array}$ & $\begin{array}{l}1.78 \mathrm{~b} \\
(2.67)\end{array}$ & $\begin{array}{l}1.32 \mathrm{~b} \\
(1.24)\end{array}$ & $\begin{array}{l}1.04 \mathrm{a} \\
(0.58)\end{array}$ & $\begin{array}{l}1.55 \mathrm{~b} \\
(1.90)\end{array}$ \\
\hline \multicolumn{2}{|c|}{$\begin{array}{c}\text { S. Em. } \pm \\
\text { C. D. at } 5 \%\end{array}$} & $\begin{array}{c}0.08 \\
\text { NS }\end{array}$ & $\begin{array}{l}0.07 \\
0.22\end{array}$ & $\begin{array}{l}0.08 \\
0.24\end{array}$ & $\begin{array}{l}0.08 \\
0.24\end{array}$ & $\begin{array}{l}0.06 \\
0.18\end{array}$ & $\begin{array}{l}0.07 \\
0.22\end{array}$ \\
\hline \multicolumn{2}{|c|}{ C. V. \% } & 11.50 & 12.02 & 14.17 & 17.22 & 14.95 & 27.85 \\
\hline $\mathbf{I} \times \mathbf{P}$ & $\begin{array}{c}\text { S. Em. } \pm \\
\text { C. D. at } 5 \%\end{array}$ & & & & & & $\begin{array}{l}0.03 \\
0.08\end{array}$ \\
\hline \multirow{2}{*}{\multicolumn{2}{|c|}{$\begin{array}{c}\text { Nitrogenous fertilizer } \\
(\mathbf{N})\end{array}$}} & \multirow{2}{*}{$\begin{array}{l}\text { Before } \\
\text { spray }\end{array}$} & \multicolumn{4}{|c|}{ Leafhoppers/leaf days after spray (DAS) } & Mean \\
\hline & & & 1 DAS & 3 DAS & 5 DAS & 7 DAS & \\
\hline \multicolumn{2}{|r|}{$\mathbf{N}_{\mathbf{0}}$} & $\begin{array}{l}2.22 \mathrm{a} \\
(4.43) \\
\end{array}$ & $\begin{array}{l}1.94 \mathrm{ab} \\
(3.26)\end{array}$ & $\begin{array}{l}1.77 \mathrm{a} \\
(2.63) \\
\end{array}$ & $\begin{array}{r}1.50 \mathrm{a} \\
(1.75) \\
\end{array}$ & $\begin{array}{c}1.30 \\
(1.19) \\
\end{array}$ & $\begin{array}{l}1.63 \mathrm{a} \\
(2.16) \\
\end{array}$ \\
\hline \multicolumn{2}{|r|}{$\mathbf{N}_{1}$} & $\begin{array}{l}2.23 \mathrm{a} \\
(4.47)\end{array}$ & $\begin{array}{l}1.89 \mathrm{a} \\
(3.07)\end{array}$ & $\begin{array}{l}1.73 \mathrm{a} \\
(2.49)\end{array}$ & $\begin{array}{l}1.48 \mathrm{a} \\
(1.69)\end{array}$ & $\begin{array}{c}1.29 \\
(1.16)\end{array}$ & $\begin{array}{l}1.60 \mathrm{a} \\
(2.06)\end{array}$ \\
\hline \multicolumn{2}{|r|}{$\mathbf{N}_{2}$} & $\begin{array}{l}2.38 \mathrm{ab} \\
(5.16)\end{array}$ & $\begin{array}{l}2.09 \mathrm{bc} \\
(3.87)\end{array}$ & $\begin{array}{l}1.87 \mathrm{ab} \\
(3.00)\end{array}$ & $\begin{array}{l}1.60 \mathrm{ab} \\
(2.06)\end{array}$ & $\begin{array}{l}1.35 \\
(1.32)\end{array}$ & $\begin{array}{l}1.73 \mathrm{ab} \\
(2.49)\end{array}$ \\
\hline \multicolumn{2}{|r|}{$\mathbf{N}_{3}$} & $\begin{array}{l}2.54 \mathrm{~b} \\
(5.95) \\
\end{array}$ & $\begin{array}{l}2.21 \mathrm{c} \\
(4.38) \\
\end{array}$ & $\begin{array}{l}2.02 \mathrm{~b} \\
(3.58) \\
\end{array}$ & $\begin{array}{l}1.71 \mathrm{~b} \\
(2.42) \\
\end{array}$ & $\begin{array}{c}1.39 \\
(1.43) \\
\end{array}$ & $\begin{array}{l}1.83 b \\
(2.85)\end{array}$ \\
\hline \multicolumn{2}{|c|}{$\begin{array}{c}\text { S. Em. } \pm \\
\text { C. D. at } 5 \% \\
\end{array}$} & $\begin{array}{l}0.08 \\
0.22\end{array}$ & $\begin{array}{l}0.07 \\
0.20\end{array}$ & $\begin{array}{l}0.06 \\
0.17\end{array}$ & $\begin{array}{l}0.06 \\
0.16\end{array}$ & $\begin{array}{l}0.05 \\
\mathrm{NS}\end{array}$ & $\begin{array}{l}0.05 \\
0.15\end{array}$ \\
\hline \multicolumn{2}{|c|}{ C. V. \% } & 12.43 & 12.86 & 12.51 & 13.68 & 13.07 & 23.92 \\
\hline $\mathbf{N} \times \mathbf{P}$ & $\begin{array}{c}\text { S. Em. } \pm \\
\text { C. D. at } 5 \%\end{array}$ & & & & & & $\begin{array}{l}0.03 \\
0.04\end{array}$ \\
\hline $\mathbf{I} \times \mathbf{N}$ & $\begin{array}{c}\text { S. Em. } \pm \\
\text { C. D. at } 5 \%\end{array}$ & & & & & & $\begin{array}{l}0.12 \\
\mathrm{NS}\end{array}$ \\
\hline
\end{tabular}

Note:

1. Figures in parentheses are retransformed values; those outside are $\sqrt{\mathrm{X}+0.5}$ value.

2. Treatment means with letter (s) in common are not significant at $5 \%$ level of significance

3. Insecticide: $\mathrm{I}_{0}=$ Control (No spray), $\mathrm{I}_{1}=$ Dimethoate $30 \mathrm{EC}, \mathrm{I}_{2}=$ Thiamethoxam $25 \mathrm{WG}, \mathrm{I}_{3}=$ Flonicamid $50 \mathrm{WP}$ and $\mathrm{I}_{4}=$ Dinotefuran $20 \mathrm{SG}$

4. Fertilizer: $\mathrm{N}_{0}=0 \mathrm{~kg} / \mathrm{ha}, \mathrm{N}_{1}=50 \mathrm{~kg} / \mathrm{ha}, \mathrm{N}_{2}=100 \mathrm{~kg} / \mathrm{ha}$ and $\mathrm{N}_{3}=150 \mathrm{~kg} / \mathrm{ha}$

5. Okra variety: Gujarat Anand Okra-5 
Table.4 Impact of insecticides and nitrogenous fertilizer on incidence of leafhopper, A. biguttula biguttula in okra (pooled over sprays) during (kharif, 2015)

\begin{tabular}{|c|c|c|c|c|c|}
\hline \multicolumn{2}{|c|}{$\begin{array}{c}\text { Treatment } \\
\text { Insecticides (I) }\end{array}$} & $\begin{array}{c}\text { Leafhoppers/ } \\
\text { leaf }\end{array}$ & \multicolumn{2}{|c|}{ Nitrogenous fertilizer $(\mathbf{N})$} & $\begin{array}{c}\text { Leafhoppers/ } \\
\text { leaf }\end{array}$ \\
\hline & $\mathbf{I}_{\mathbf{0}}$ & $2.36 \mathrm{~d}(5.09)$ & \multicolumn{2}{|c|}{$\mathbf{N}_{\mathbf{0}}$} & $1.58(1.99)$ \\
\hline & $\mathbf{I}_{1}$ & $1.74 \mathrm{c}(2.52)$ & \multicolumn{2}{|c|}{$\mathbf{N}_{1}$} & $1.65(2.23)$ \\
\hline & $\mathbf{I}_{2}$ & $1.65 \mathrm{c}(2.23)$ & \multicolumn{2}{|c|}{$\mathbf{N}_{2}$} & $1.63(2.16)$ \\
\hline & $\mathbf{I}_{3}$ & $1.07 \mathrm{a}(0.65)$ & \multicolumn{2}{|c|}{$\mathbf{N}_{3}$} & $1.73(2.50)$ \\
\hline \multicolumn{2}{|c|}{$\mathbf{I}_{4}$} & $1.41 \mathrm{~b}(1.49)$ & & & \\
\hline S. Em & C. D. at $5 \%$ & 0.070 .21 & \multicolumn{2}{|c|}{ S. Em. \pm C. D. at 5\% } & $0.05 \mathrm{NS}$ \\
\hline \multicolumn{2}{|c|}{ C. V. \% } & 22.88 & \multicolumn{2}{|c|}{ C. V.\% } & 22.08 \\
\hline $\mathbf{I} \times \mathbf{P}$ & $\begin{array}{c}\text { S. Em. } \pm \\
\text { C. D. at } 5 \%\end{array}$ & $\begin{array}{l}0.04 \\
0.13\end{array}$ & $\mathbf{N} \times \mathbf{P}$ & $\begin{array}{c}\text { S. Em. } \pm \\
\text { C. D. at } 5 \%\end{array}$ & $0.02 \mathrm{NS}$ \\
\hline \multirow[t]{2}{*}{$\mathbf{S} \times \mathbf{I}$} & $\begin{array}{c}\text { S. Em. } \pm \\
\text { C. D. at } 5 \%\end{array}$ & $\begin{array}{l}0.05 \\
0.16 \\
\end{array}$ & $\mathbf{S} \times \mathbf{N}$ & $\begin{array}{c}\text { S. Em. } \pm \\
\text { C. D. at } 5 \%\end{array}$ & 0.050 .13 \\
\hline & & & $\mathbf{I} \times \mathbf{N}$ & $\mathbf{I} \times \mathbf{N}$ & $0.06 \mathrm{NS}$ \\
\hline
\end{tabular}

\section{Note:}

1. Figures in parentheses are retransformed values; those outside are $\sqrt{\mathrm{X}+0.5}$ value.

2. Treatment means with letter (s) in common are not significant at $5 \%$ level of significance

3. Insecticide: $\mathrm{I}_{0}=$ Control (No spray), $\mathrm{I}_{1}=$ Dimethoate $30 \mathrm{EC}, \mathrm{I}_{2}=$ Thiamethoxam $25 \mathrm{WG}, \mathrm{I}_{3}=$

Flonicamid $50 \mathrm{WP}$ and $\mathrm{I}_{4}=$ Dinotefuran $20 \mathrm{SG}$

4. Fertilizer: $\mathrm{N}_{0}=0 \mathrm{~kg} / \mathrm{ha}, \mathrm{N}_{1}=50 \mathrm{~kg} / \mathrm{ha}, \mathrm{N}_{2}=100 \mathrm{~kg} / \mathrm{ha}$ and $\mathrm{N}_{3}=150 \mathrm{~kg} / \mathrm{ha}$

5. Okra variety: Gujarat Anand Okra-5

Table.5 Impact of nitrogenous fertilizer and insecticides on fruit yield of okra (kharif, 2015)

\begin{tabular}{|c|c|c|c|c|}
\hline \multicolumn{2}{|c|}{ Insecticides } & Yield (q/ha) & Fertilizer & Yield (q/ha) \\
\hline \multicolumn{2}{|c|}{$\mathbf{I}_{0}$} & $42.47 \mathrm{~d}$ & $\mathrm{~N}_{\mathbf{0}}$ & $52.95 \mathrm{~b}$ \\
\hline \multicolumn{2}{|r|}{$I_{1}$} & $53.63 c$ & $\mathbf{N}_{1}$ & $56.25 b$ \\
\hline \multicolumn{2}{|r|}{$\mathbf{I}_{2}$} & $61.19 b$ & $\mathbf{N}_{2}$ & $61.82 \mathrm{a}$ \\
\hline \multicolumn{2}{|r|}{$\mathbf{I}_{3}$} & $71.96 \mathrm{a}$ & $\mathbf{N}_{3}$ & $62.37 \mathrm{a}$ \\
\hline \multicolumn{2}{|r|}{$\mathbf{I}_{4}$} & $62.69 \mathrm{~b}$ & & \\
\hline \multicolumn{5}{|c|}{ ANOVA } \\
\hline \multicolumn{2}{|c|}{$\begin{array}{c}\text { S. Em. } \pm \\
\text { C. D. at } 5 \%\end{array}$} & $\begin{array}{l}2.28 \\
7.11\end{array}$ & $\begin{array}{c}\text { S. Em. } \pm \\
\text { C. D. at } 5 \%\end{array}$ & $\begin{array}{l}1.72 \\
4.97\end{array}$ \\
\hline \multicolumn{2}{|c|}{ C.V \% } & 13.53 & C.V. \% & 11.42 \\
\hline I $\times N$ & $\begin{array}{c}\text { S. Em. } \pm \\
\text { C. D. at } 5 \%\end{array}$ & \multicolumn{3}{|c|}{$\begin{array}{l}3.85 \\
\mathrm{NS}\end{array}$} \\
\hline
\end{tabular}

\section{Note:}

1. Insecticide: $\mathrm{I}_{0}=$ Control (No spray), $\mathrm{I}_{1}=$ Dimethoate $30 \mathrm{EC}, \mathrm{I}_{2}=$ Thiamethoxam $25 \mathrm{WG}, \mathrm{I}_{3}=$ Flonicamid $50 \mathrm{WP}$ and $\mathrm{I}_{4}=$ Dinotefuran $20 \mathrm{SG}$

2. Fertilizer: $\mathrm{N}_{0}=0 \mathrm{~kg} / \mathrm{ha}, \mathrm{N}_{1}=50 \mathrm{~kg} / \mathrm{ha}, \mathrm{N}_{2}=100 \mathrm{~kg} / \mathrm{ha}$ and $\mathrm{N}_{3}=150 \mathrm{~kg} / \mathrm{ha}$

3. Okra variety: Gujarat Anand Okra-5 
More or less, similar trend was also found at 3 DAS. The leafhopper population recorded at 5 DAS showed that significantly the lowest (0.40) population was recorded in the plots treated with flonicamid $50 \mathrm{WP} 0.015 \%$. The dinotefuran 20 SG $0.008 \%$ (1.24) was at par with dimethoate 30 EC $0.03 \%$ (1.84) followed by thiamethoxam 25 WP $0.01 \%$ (2.12). The highest 5.45 leafhopper population was observed in control (no spray) plots. At 7 DAS, flonicamid 50 WP $0.015 \%$ (0.35) was found significantly superior over the tested insecticides. However, it was at par with $\begin{array}{lllll}\text { dinotefuran } 20 & \text { SG } & 0.008 \% & (0.58) \text {. }\end{array}$ Thiamethoxam 25 WP $0.01 \% \quad(0.99)$ and dimethoate 30 EC $0.03 \%$ (1.11) were at par and stood second next in order for the control of leafhopper in okra. The highest (4.38) leafhopper population was observed in control treatment (no spray).

Pooled over period of mean data (Table 3) indicated that flonicamid 50 WP $0.015 \%$ exhibited significantly superior than all the tested insecticides by recording the lowest (0.82 leafhoppers/leaf) population of leafhopper. Dinotefuran $20 \mathrm{SG}$ at $0.008 \%$ (1.90), thiamethoxam $25 \mathrm{WP}$ at $0.01 \%(2.42)$ and dimethoate $30 \mathrm{EC}$ at $0.03 \%$ (2.42) were statistically at par with each other. The highest (5.12) leafhopper population was observed in control plots.

\section{Independent effect of fertilizer $(\mathbf{N})$}

The data on impact of nitrogenous fertilizer at different doses on incidence of leafhopper are depicted in Table 3. The leafhopper activity was found uniform in all the treatments before treatment as ANOVA was non-significant. At 1 DAS, minimum population was observed in $\mathrm{N}_{1}$ (3.07 leafhoppers/leaf) and it was at par $\mathrm{N}_{0}$ (3.26) and $\mathrm{N}_{2}$ (3.87) which were at par with $\mathrm{N}_{3}$ (4.38). At 3 DAS, minimum population was observed in $\mathrm{N}_{0}$ (2.63) and it was at par with $\mathrm{N}_{1}$ (2.49) and $\mathrm{N}_{2}$ (3.00). The higher population was recorded in $\mathrm{N}_{3}$ (3.58). At 5 DAS, more or less similar trend was observed. Non-significant effect of nitrogenous fertilizer was observed at 7 DAS.

The periodic data on leafhopper were also pooled and are presented in Table 3 . Significantly lower (2.06 leafhoppers/leaf) population was recorded in $\mathrm{N}_{1}$ i.e. $50 \mathrm{~kg} / \mathrm{ha}$. However, it was at par with control (no fertilizer) and $\mathrm{N}_{2}(100 \mathrm{~kg} / \mathrm{ha})$. The highest population (2.82) was recorded in the highest dose of nitrogen $\left(\mathrm{N}_{3}\right)$. Although, it was at par with $\left(\mathrm{N}_{2}\right)$.

Interactive effect of insecticide and fertilizer $(\mathbf{I} \times \mathbf{N})$

The interactive effect of insecticides with different doses of nitrogenous fertilizer on okra crop showed (Table 3) non-significant impact on population of $A$. biguttula biguttula.

\section{Pooled over sprays}

Pooled over sprays data presented in Table 4 indicated that flonicamid 50 WP $0.015 \%$ recorded the lowest (0.65 leafhoppers/leaf) leafhopper population than rest of the insecticides. The second best insecticide was dinotefuran 20 SG $0.008 \%$ and recorded 1.49 per leaf. Thiamethoxam 25 WP $0.01 \%$ (2.23) and dimethoate 30 EC $0.03 \%$ (2.52) also showed better effectiveness against leafhopper population by remaining at par with each other. The highest (5.09) leafhopper population was observed in control plots.

Misra (2002) reported that imidacloprid and thiamethoxam both used at $25 \mathrm{~g}$ a.i./ha proved significantly superior in controlling aphid and leafhopper in okra. Flonicamid was found most effective insecticide against major sap sucking insects like aphid, leafhopper, whitefly, scales, thrips and mealybugs on 
vegetable and potatoes (Kodandaram et al., 2010). Thiamethoxam $20 \mathrm{~g}$ a.i/ha and fipronil $50 \mathrm{~g} \mathrm{a.i/ha}$ effectively reduced the sucking pests viz., leafhopper, whitefly and red spider mite in okra (Mohansundaram and Sharma, 2011). Thus, the present findings are in agreement with the earlier reports.

The pooled data (Table 4) revealed that different doses of nitrogenous fertilizer had non-significant impact on population development of leafhopper. The interaction between different dose of nitrogenous fertilizer and insecticides $(I \times N)$ could not impose any significant effect on leafhopper population.

The population of leafhopper (A. devastans) was significantly increased with increasing nitrogen levels (Razaq et al., 2014). Baloch et al., (2013) results showed that the highest population of leafhopper $(1.05 \pm 0.66)$ was recorded on okra plants treated with $\mathrm{Di}$ ammonium Phosphate followed by urea + DAP, control (No fertilizers) and urea alone. Thus, present findings are more or less similar to previous findings.

\section{Yield}

\section{Impact of insecticides (I)}

Green okra fruit was recorded in all treatments and control during kharif 2015 and are presented in Table 5. All the treatments registered significantly higher yield of green okra fruits than control. Plots treated with flonicamid 50 WP $0.015 \%$ yielded significantly the highest (71.96 q/ha) and proved to be most effective. Dinotefuran 20 SG $0.008 \%$ and thiamethoxam 25 WP $0.01 \%$ (61.19 q/ha) also yielded higher and differed significantly with each other. Dimethoate 30 EC at $0.03 \%$ produced significantly the lowest fruit yield $(53.63 \mathrm{q} / \mathrm{ha})$ and proved to be least effective. Control treatment (no spray) recorded the lowest fruit yield (42.47 q/ha).

\section{Impact of nitrogenous fertilizer (N)}

The data on impact of nitrogenous fertilizer as independent variable on green okra fruit yield are depicted in Table 5. The highest green okra fruit yield $(62.37 \mathrm{q} / \mathrm{ha})$ was recorded in $\mathrm{N}_{3}$ (150 kg N/ha) which was statistically at par with $\mathrm{N}_{2}(61.83 \mathrm{q} / \mathrm{ha})$. Among the different doses of nitrogenous fertilizers, $\mathrm{N}_{1}$ recorded $56.25 \mathrm{q} / \mathrm{ha}$ green fruit okra yield and was statistically at par with green fruit yield of okra in $\mathrm{N}_{0}(52.95 \mathrm{q} / \mathrm{ha})$.

\section{Interaction of different dose of nitrogenous fertilizer and insecticides $(\mathbf{I} \times \mathbf{N})$}

The interaction effect of insecticides and nitrogenous fertilizer showed non-significant results. Scanty information is available so far effect of nitrogenous fertilizer on the effectiveness of insecticides against leafhopper is concerned in okra crop. Echezona et al., (2010) reported that the higher okra fruit yield was recorded by combining of $1.0 \mathrm{~kg} / \mathrm{ha}$ carbofuran with $80 \mathrm{~kg}$ $\mathrm{N} / \mathrm{ha}$, while, the best economic return was achieved by combining of $0.5 \mathrm{~kg} / \mathrm{ha}$ carbofuran with $80 \mathrm{~kg} \mathrm{~N} / \mathrm{ha}$. Under the present investigation, more or less similar trend was found. Sylvester et al., (2014) concluded that complementary application of poultry manure (10 tones/ha) and NPK (300 $\mathrm{kg} / \mathrm{ha}$ ) favoured okra growth and yield, most in terms of quantity and quality and decreased the infestation of pest. The contradictory results noticed might be attributed due to poultry manure given as NPK.

\section{References}

Anonymous, 2014. National Horticulture Board Database (www.agriexchange. apeda.gov.in) 
Baloch, N., Lanjar, A. G., Samo, M. A., Solangi, A. W. and Khuhro, S. A. 2013. Impact of fertilizer application on insect pest population in okra, Abelmoschus esculentus L. crop. Sindh University Research Journal, 45 (2): 247-252.

Echezona, B. C., Asiegbu, J. E. and Izuagba, A. A. 2010. Flee beetle population and economic yield of okra as influenced by nitrogen and 2, 3-Dihydro-2, 2Dimethyl Benzofuran. African Crop Science Journal, 18 (3): 97 - 105.

Kodandaram, M. H., Rai, A. B. and Halder, J. 2010. Novel insecticides for the management of insect pests in vegetable crops: A review. Vegetable Science, 37 (2): 109-123.

Krishnaiah, K., 1980. Methodology for assessing crop losses due to pests of vegetable. Assessment of crop losses due to pests and diseases. Proc. of Workshop held from Sept, 19-30, 1977 at U.A.S., Bangalore, and pp. 259-267.

Misra, H. P., 2002. Field evaluation of some newer insecticides against aphid (Aphis gossypii) and jassids (Amrasca biguttula biguttula) on okra. Indian Journal of Entomology, 64 (1): 80-84.

Mohansundaram, A., and Sharma, R. K. 2011. Abundance of pest complex of okra in relation to abiotic and biotic factors. Ann. Pl. Protec. Sci., 19 (2): 286-290.

Pal, S., Maji, T. B. and Mondal, P. (2013). Incidence of insect pest on okra Abelmoschus esculentus (L) Moench in red lateritic zone of West Bengal.
Journal of Plant Protection Sciences, 5 (1): 59-64.

Rawat, R. R., and Sadhu, H. R. 1973. Estimation of losses in growth and yield of okra due to Empoasca devastans (dist). and Earias spp. Indian J. Ent., 35: 252-254. [Fide: Sridharan, S., Sekhar, K. C. and Ramakrishnan, N. (2015). Effect of mineral oil and its combination against leafhopper, Amrasca biguttula biguttula in okra. Indian Journal of Plant Protection, 43 (2): 133-142].

Razaq, M., Haneef, Q., Athar, H. R., Nasir, M. and Afzal, M. 2014. Interactive effect of nitrogen and insecticide on jassid, Amrasca devastans (Dist.) population and photosynthetic capacity of okra Abelmoschus esculentus (L.) Moench. Pakistan Journal of Zoology, 46 (2): 577-579.

Sridharan, S., Sekhar, K. C. and Ramakrishnan, N. 2015. Effect of mineral oil and its combination against leafhopper, Amrasca biguttula biguttula in okra. Indian Journal of Plant Protection, 43 (2): 133-142.

Sylvester, R. A., Nuga, B. O., Ndowa E. S., Lale and Osayi, R. N. 2014. Effect of organic and inorganic fertilizers on okra (Abelmoschus esculentus L. Moench) production and incidence of insect pests in the humid tropics. IOSR Journal of Agricultural Veterinary Science, 7 (4): 2319-2372.

\section{How to cite this article:}

Naziya P. Pathan, T.M. Bharpoda, D.B. Sisodiya and Gohel, N.M. 2017. Effect of Nitrogenous Fertilizer and Insecticides on Incidence of Leafhopper, Amrasca biguttula biguttula (Ishida) in Kharif Okra. Int.J.Curr.Microbiol.App.Sci. 6(10): 540-550. doi: https://doi.org/10.20546/ijcmas.2017.610.066 